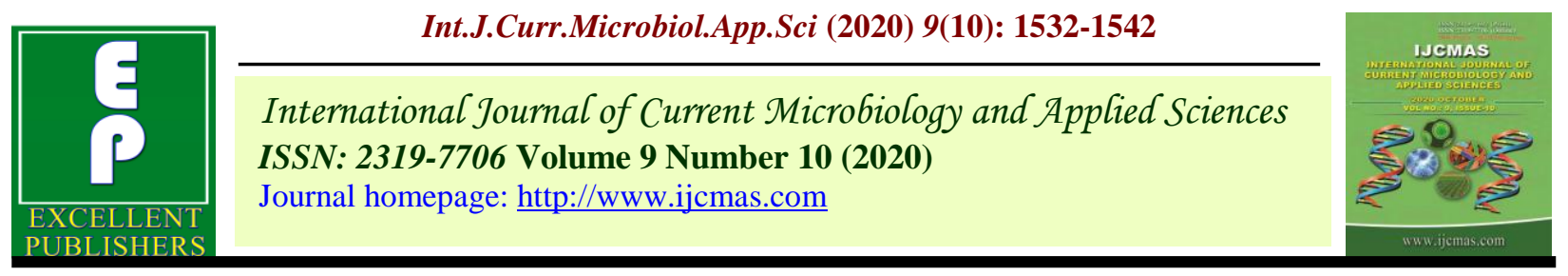

Review Article

https://doi.org/10.20546/ijcmas.2020.910.183

\title{
Cryopreservation of Fruit Genetic Resources-A Review
}

\author{
P. L. Anushma ${ }^{1 *}$, K. Dhanyasree ${ }^{2}$ and M. Rafeekher ${ }^{2}$ \\ ${ }^{1}$ ICAR-Indian Institute of Horticultural Research, Bengaluru, Karnataka, India \\ ${ }^{2}$ College of Agriculture, Vellayani, Kerala, India \\ *Corresponding author
}

\section{A B S T R A C T}

\section{Keywords}

Cryopreservation,

Fruit genetic

resources

Article Info

Accepted:

12 September 2020

Available Online:

10 October 2020
Conserving the plant genetic resources through any means is of utmost importance to prevent the loss of plant biodiversity. Long-term storage using liquid nitrogen (LN) is usually adopted for developing long-term base collections of crops for use in the distant future. The fruit crops are commonly propagated through vegetative means and are majorly conserved as ex situ field gene banks, which require large areas and demand intensive field management. The need for repeated reculturing and chances of contamination during subcultures in in vitro banking necessitate a long-term contamination free conservation through cryobanking. Improved cryopreservation techniques will provide added security in the preservation of important woody plant germplasm formerly preserved only as field collections.

\section{Introduction}

Conserving the plant genetic resources through any means is of utmost importance to prevent the loss of plant biodiversity. Conservation can be achieved through two broad methods, i.e., in situ and ex situ. Conservation of germplasm under natural conditions is referred to as in situ conservation. The ex situ conservation refers to preservation of germplasm in gene banks. Through ex situ conservation techniques, it is possible to preserve entire genetic diversity of a crop species at one place, with comparatively lesser expense and less intensive management. The ex situ conservation methods comprise of seed gene banks, field gene banks, botanic gardens, in vitro banks, cryogene bank and DNA banks. Long-term storage using liquid nitrogen (LN) is usually adopted for developing long-term base collections of crops for use in the distant future, functioning as a safety backup for similar collections held in clonal repositories, field gene banks (FGB) or in situ conserved genetic material is subject to threats from natural vagaries.

The cryogene banks (preserving the genetic resources in ultra-low) temperatures helps in long-term conservation (for indefinite periods theoretically) of plant genetic resources which is very easier and require limited space and management. 


\section{Cryopreservation}

The term cryopreservation is used for the process of storage of living material at ultralow temperatures in liquid nitrogen $(-196$ $\left.{ }^{\circ} \mathrm{C}\right)$ or its vapour phase $\left(-150{ }^{\circ} \mathrm{C}\right)$. At this temperature, all cellular, metabolic and biochemical events stop, and the plant material can be stored without any changes or deterioration for extended time periods (Reed, 2017). The principle of cryopreservation is to bring plants cells or tissues to a zero metabolism and non-dividing state by reducing the temperature in presence of cryoprotectant.

The ultra-low temperature storage of plant genetic resources can be achieved by keeping them over solid carbon dioxide $\left(-79^{\circ} \mathrm{C}\right)$, in low temperature deep freezers $\left(-80^{\circ} \mathrm{C}\right)$, in vapour phase nitrogen $\left(-150^{\circ} \mathrm{C}\right)$ or in liquid nitrogen $\left(-196^{\circ} \mathrm{C}\right)$. However, liquid nitrogen is most widely used in long term storages owing to its chemically inert and nontoxic nature, low cost, easy availability and due to noninflammable properties.

\section{Relevance of cryopreservation in conserving fruit genetic resources}

The fruit crops are commonly propagated through vegetative means and are majorly conserved as ex situ field gene banks. These field gene banks require large areas to conserve the genetically variable germplasm and demand intensive field management. Being highly heterogeneous and recalcitrant in nature, the fruit crops could not be conserved through seed banking. In addition, the field gene banks are always in the threat from pest and disease outbreak, changing climate and natural calamities. The short- and medium-term conservation through in vitro banking back up the clonal repositories, the need for repeated reculturing and chances of contamination during subcultures necessitate a long-term contamination free conservation through cryobanking.

\section{Selection of plant material}

Cryopreservation involves storage of plant material such as intermediate or recalcitrant seeds (which are difficult to conserve through other techniques), dormant buds from temperate and subtropical fruit crops which undergo winter dormancy, shoot tips or meristems, zygotic or somatic embryos and pollen at ultra-low temperatures. The ideal plant material for cryopreservation should have attributes such as smaller size, less in moisture content, highly vacuolated cells and should be collected from healthy younger parts. Meristems are proven to be ideal explant for cryopreservation (Kalaselvi et al., 2017)

\section{Mechanism of cryopreservation}

Cryopreservation of biological tissues can be successful only when intracellular ice crystals do not form, since they cause irreversible damage to cell membranes and thus destroy their semipermeability. Crystal formation without an extreme reduction of cellular water can be prevented by vitrification, i.e., the non crystalline solidification of water. Vitrification is a physical process by which a highly concentrated cryoprotective solution supercools to very low temperatures and on further cooling solidifies into a metastable glass, without undergoing crystallization.

As glass is exceedingly viscous and stops all chemical reactions that require molecular diffusion, its formation leads to metabolic inactivity and stability over time. To achieve a state of vitrification, there are two conditions required: (1) rapid freezing rates and (2) a concentrated cellular solution. The latter is obtained through air drying, freeze dehydration, application of penetrating or 
non-penetrating substances (cryoprotectants) or acclimation (Agrawal et al., 2019)

\section{Techniques of cryopreservation}

From the original slow cooling approach, research has moved to easier and more reproducible techniques such as desiccation, pregrowth, pregrowth-desiccation, vitrification (Sakai et al., 1990), encapsulation-dehydration (Fabre and Dereuddre, 1990), encapsulation-vitrification (Matsumoto et al., 1994), droplet-freezing (Schäfer-Menuhr et al., 1994) and ultrarapid freezing or droplet-vitrification (Agrawal et al., 2004 and Panis et al., 2005). Most recent protocols are ones using an aluminium cryoplate or stainless steel cryo-meshes, which combines the methods of encapsulation-vitrification and dropletvitrification (Funnekotter et al., 2017 and Matsumoto, 2017).

\section{Slow Freezing or Controlled Freezing (Classical Method)}

Slow cooling comprises steps of tissue dehydration followed by slow cooling (at rates of 0.1 to $4^{\circ} \mathrm{C} / \mathrm{min}$ ) of the explant, down to a defined prefreezing temperature (usually of -30 to $-40{ }^{\circ} \mathrm{C}$ ) and rapid immersion in $\mathrm{LN}$ (Engelmann, 2004). As such, cells and the external medium initially supercool, followed by extracellular ice formation and avoidance of intracellular ice formation (Mazur, 1984). Slow freezing requires the use of costly programmable freezing apparatus (with greater temperature control). This method combines the application of penetrating cryoprotective substances such as dimethyl sulfoxide (DMSO) and controlled freeze dehydration. A modification of this method is known as the two-step cooling, where no special programmable freezer is required. Plant tissues are treated with cryoprotectants, normally at room temperature $\left(25{ }^{\circ} \mathrm{C}\right)$, and are kept at $-30{ }^{\circ} \mathrm{C}$ for $30-120 \mathrm{~min}$. They are then immersed in $\mathrm{LN}$ thereafter. Cryopreserved tubes are warmed using hot water $\left(40{ }^{\circ} \mathrm{C}\right)$ for $1 \sim 2 \mathrm{~min}$, and cryoprotectants are removed from a tube. After rewarming, samples are moved from the cryotube and recultured (Agrawal et al., 2019).

\section{New Vitrification-Based Techniques}

In these techniques, vitrification is achieved by direct immersion in LN without the freeze concentration step (as in slow cooling), by exposing the cells to extremely concentrated (7 to $8 \mathrm{M}$ ) cryoprotectant solutions (Sakai and Engelmann, 2004).

\section{Dehydration freezing}

Cryopreservation of seeds of Citrus species after desiccation was attempted by Kaya et $a l$. , (2016) in Turkey, wherein the seeds of six citrus species viz., Citrus limon (L.) Burm, $C$. limonia Osbeck, $C$. jambhiri Lush., $C$. aurantium L., Poncirus trifoliata Raf. $\times C$. sinensis Osb. (Troyer citrange) and Fortunella margarita (Lour.) Swingle were tested for their initial moisture content, viability and germination. These seeds were subjected to air desiccation for different durations (depending on their initial moisture contents) so that the seed moisture content reached to 10-20 per cent. The dehydrated seeds were directly immersed into liquid nitrogen. The fresh seed moisture content was higher in Troyer citrange $(56.3 \%)$, C. limon (51.1\%) and C. jambhiri (49.7\%), while lowest was in Fortunella margarita (25.1\%). Maximum tolerance to cryopreservation was observed in $C$. jambhiri with 93.3 per cent germination after 12 hours of dehydration to moisture content of 21.1 per cent. Lowest seed germination $(43.3 \%)$ was observed in Fortunella margarita when dehydrated for 3 hours to a moisture content of 24.2 per cent, 
while the species had a better germination when desiccated for six hours to moisture content of 19.0 per cent. In other species also, the optimum seed moisture content and dehydration duration were optimized for successful cryopreservation. Thus in Citrus species, which produce polyembryonic nonorthodox oily seeds, air desiccation of seeds to moisture levels of around 20 per cent proved better for successful cryopreservation.

In Passiflora edulis, the seeds with initial moisture content of 25 per cent were desiccated to various moisture levels $(15,10$ and $5 \%$ ) by thin layer drying at $40{ }^{\circ} \mathrm{C}$ for different durations (42, 70 and 100 minutes respectively and exposed to liquid nitrogen treatment (Generoso et al., 2019). The cryopreserved seeds with water content of $10 \%$ produced all normal seedlings under in vitro while non-desiccated seeds did not produce normal seedlings. The non-desiccated seeds produced either abnormal seedling $(7.5 \%)$ or remained as ungerminated embryos $(92.5 \%)$.

\section{Encapsulation-Dehydration}

The technique involves immersion of explants in alginate solution (usually 1-5\%) and their subsequent release into a complexing agent (50-100 mM CaCl2 solution) where bead hardening occurs in 20-30 min. These synseeds are next cultured in a highly concentrated sucrose solution $(0.7-1.5 \mathrm{M})$, followed by physical dehydration to a moisture content of $20-30 \%$, using air of laminar flow or silica gel (Engelmann, 2004). The dehydrated beads are then cryopreserved. Culture of explants on sucrose-enriched medium $(0.3-0.7 \%)$ prior to encapsulation usually improves survival after desiccation and freezing. This method is simple, but somewhat labour intensive, and some species do not tolerate the high sucrose concentrations employed. The use of anti- oxidant and anti-stress compounds (e.g. lipoic acid, glutathione, glycine betaine, polyvinyl pyrrolidone, melatonin) has been shown to increase post-thaw recovery in explants cryopreserved by this method (Uchendu et al., 2010).

In rough lemon (Citrus jambhiri), the embryonic axes from three genotypes IPS-7, IPS-85 and IPS-120 were subjected to encapsulation. The fresh beads were precultured in various concentrations of sucrose $(0.3,0.5$ and $0.7 \mathrm{M})$ for 40 hours and moisture contents were recorded. Further the embryonic axes were air desiccated for 6 hours so that the moisture contents were reduced to less than 22 per cent in all the accessions. Preculturing beads on $0.5 \mathrm{M}$ sucrose followed by $6 \mathrm{~h}$ of air desiccation gave maximum survival of axes before and after cryopreservation in IPS-7 and IPS- 85, but for accession IPS-120 successful cryopreservation with a highest viability of 66.66 and 50 per cent respectively, was achieved for axes precultured for $40 \mathrm{~h}$ in 0.3 $M$ sucrose preculture media, followed by $6 \mathrm{~h}$ of desiccation. More than 100 accessions of C. jambhiri have been cryostored in the national cryobank at NBPGR, New Deli (Rohini et al., 2016).

\section{Vitrification}

In this technique, most or all freezable water is removed by physical or osmotic dehydration of explants (thus increasing cellular viscosity), followed by ultrarapid freezing which results in vitrification of intracellular solutes. A number of steps are involved - preculture of explants on medium fortified with cryoprotective substances, treatment with a loading solution (e.g. a mixture of $2 \mathrm{M}$ glycerol and $0.4 \mathrm{M}$ sucrose), dehydration with a highly concentrated vitrification solution (from 15 min up to $2 \mathrm{~h}$ ), rapid freezing and thawing, removal of 
cryoprotectants and recovery. A widely used vitrification solution (mixture of penetrating and non-penetrating cryoprotectant substances) developed by Sakai et al., (1990) is named plant vitrification solution 2 (PVS2). It consists of $30 \%(\mathrm{w} / \mathrm{v})$ glycerol, $15 \%(\mathrm{w} / \mathrm{v})$ ethylene glycol and $15 \%(\mathrm{w} / \mathrm{v})$ DMSO in liquid medium with $0.4 \mathrm{M}$ sucrose and a total molarity of $7.8 \mathrm{M}$. Cells are osmotically dehydrated by PVS2 at a nonfreezing temperature, ranging from 0 to $25{ }^{\circ} \mathrm{C}$ (Sakai et al., 1990).

Vitrification of shoot apices of pineapple in vitro plantlets was attempted by GonzalezArnao et al., (1998) wherein the apices were precultured in medium containing $0.3 \mathrm{M}$ sucrose and loaded in medium with $0.75 \mathrm{M}$ sucrose $+1 \mathrm{M}$ glycerol for 25 minutes. When the apices were dehydrated in PVS2 solution under different exposure periods at $25^{\circ} \mathrm{C}$, the survival after cryopreservation was very less.

When the apices were dehydrated in PVS2 at $0^{\circ} \mathrm{C}$, the apices survived better. The highest survival of apices after cryopreservation (65\%) was observed with PVS2 vitrification solution at $0^{\circ} \mathrm{C}$ for 7 hours before rapid freezing in liquid nitrogen. The study has emphasized on the relevance vitrification temperature on successful cryopreservation of pineapple shoot apices.

In Passiflora pohlii, a wild species of Passiflora resistant to soil borne pathogens, studies to cryopreserve the nodal segments of $0.5 \mathrm{~cm}$ through vitrification were taken up. The explants after preculture in half strength MS media containing $0.7 \mathrm{M}$ sucrose, were exposed to two vitrification solutions namely PVS2 (30\% glycerol, 15\% dimethyl sulfoxide (DMSO), $15 \%$ ethylene glycol (EG) and $13.7 \%$ sucrose, w/v) and PVS3 (50\% glycerol and $50 \%$ sucrose, w/v) for different exposure timings before immersing to liquid nitrogen. The highest recovery $(65 \%)$ was obtained with the vitrification technique using treatment with the PVS3 solution for 30 to $120 \mathrm{~min}$. Also, 40 per cent recovery was observed in segments treated with PVS2 for 60 minutes when incubated in dark conditions while post-warming incubation in light did not recover any cryopreserved nodal segments (Merhy et al., 2014).

\section{Encapsulation-vitrification}

In this technique, the explants are encapsulated and subjected to vitrification, without any further physical desiccation. It combines the advantages of vitrification and of encapsulation-dehydration (ease of manipulation of encapsulated explants) and applied to apices of an increasing number of species (Matsumoto et al., 1994 and Sakai and Engelmann, 2007)

In pineapple varieties MD-2 and Peurto Rico, efficiency of encapsulation-vitrification of shoot apices was compared with vitrification by Gamez-Pastrana et al., (2004). To improve the survival of apices on cryostorage, the pretreatment conditions were modified by supplementing Proline (3 M) along with Sucrose. Optimal conditions involved the encapsulation of pineapple apices in calcium alginate $(3 \%)$ followed by two-stage preculture in liquid medium with $0.16 \mathrm{M}$ sucrose $+0.3 \mathrm{M}$ proline for $24 \mathrm{~h}$ and then transfer to $0.3 \mathrm{M}$ sucrose $+0.3 \mathrm{M}$ proline for an additional 24 hours.

Encapsulation-vitrification was more effective for cryopreserving apices of both varieties when using PVS3 for dehydration. These conditions gave the highest survival rates after cryopreservation (54\% with apices of MD-2 and $83 \%$ with apices of Puerto Rico) while dehydration with PVS2 resulted in lesser survival of encapsulated beads as compared to vitrification 


\section{Droplet-Freezing/Vitrification}

Droplet-freezing method (also referred as ultra-freezing or fast-freezing method in literature) was initially established successfully for potato (Schäfer-Menuhr et al., 1994). The method refers to refers to droplets of cryoprotectant on an aluminium foil, into which explants are placed for cooling and rewarming. In this method, a drop of PVS2 is placed on aluminium foil strips, onto which explants are placed and plunged in LN. The application of droplet-freezing allows higher cooling and thawing rates compared to normal vitrification (of about $130^{\circ} \mathrm{C} / \mathrm{min}$ ), and the chance to obtain a vitrified state during freezing and to avoid devitrification during thawing increases. Varied explants such as shoot tips, meristems, embryogenic callus, hairy roots and embryonic axes have been used.

In banana, explants used for cryopreservation of banana (Musa L. spp.) are mainly sourced from tissue culture. The regenerative potential of cryopreserved sucker derived meristem (SM) was compared with two types of routinely employed explants viz., in vitroraised single-shoot meristems (IVM) and proliferating meristems (PM). The regeneration frequency of SM was high $(60.0 \pm 11.5 \%)$ and statistically comparable to PM $(68.3 \pm 4.4 \%)$ and IVM $(55.6 \pm 11.1 \%)$ after using the droplet vitrification cryopreservation technique.

The total time required for cryopreserving plants from SM (2 months) was substantially less than that for PM (14 months) and IVM (8 months). In addition, the study revealed that cryopreserved plants were statistically comparable to the mother plants raised from suckers for all important growth and yield parameters. Thus Musa germplasm could be effectively cryopreserved using droplet vitrification method with a new type of explant, the sucker derived meristem (Agrawal et al., 2014).

In Citrus limon, successful cryopreservation of in vitro grown shoot tips through droplet vitrification was reported in two cultivars 'Frost Eureka limon' and 'Cook Eureka limon' by Yi et al., (2018), wherein preculturing shoot tips in MS + 0.3 M sucrose for 48 hours and then treated in MS + 0.5 M sucrose for 16 hours was optimized, followed by loading and dehydration. The shoot tips were treated with two vitrification solutions PVS2 and PVS3 for varied exposure times (30, 60, 90 and 120 minutes) at $0{ }^{\circ} \mathrm{C}$ and transferred to aluminium foil strips containing PVS2 or PVS3 and directly plunged into liquid nitrogen. In both the cultivars, higher regrowth percentages of shoot apices on micrografting on Troyer citrange seedlings were observed for PVS2 treatment for 60 minutes or PVS3 for 90 minutes.

\section{Cryoplates method}

Recent developments in cryopreservation of plants have been steps that have replaced use of aluminium foil with aluminium cryo-plates (V cryo-plate and D cryo-plate, Matsumoto, 2017). In these methods, shoot tips are stuck on special aluminium plates with uniform wells using alginate, and subjected to vitrification procedures. These techniques have two main advantages; (1) they are userfriendly because samples are easy to handle, with reduced risk of damage or loss of explants, and (2) they have a high rate of regrowth owing to the very high cooling and warming rates (Niino et al., 2013; Yamamoto et al., 2015 and Funnekotter et al., 2017). The $\mathrm{V}$ cryo-plate method (Yamamoto et al., 2011b) is based on PVS2-vitrificationdehydration of explants on a cryo-plate, and the D cryo-plate method (Niino et al., 2013) is based on air dehydration. Precultured (0.3 M sucrose) shoot tips are attached to small 
wells of a cryo-plate with alginate beads and treated with LS solution (2 M glycerol + 0.6$1 \mathrm{M}$ sucrose) for 15-30 $\mathrm{min}$ and then dehydrated with PVS2 solution (V cryo-plate method) or in a laminar flow cabinet (D cryopate method) for a suitable duration. Then the cryoprotected and dehydrated shoot tips are immersed in LN directly. For regrowth, shoot tips attached to the cryo-plate are transferred to a $1 \mathrm{M}$ sucrose solution for rapid warming and unloading at $25{ }^{\circ} \mathrm{C}$ for $15 \mathrm{~min}$ and plated on a culture medium. Higher rates of regrowth have been reported using the cryoplate methods due to the very high cooling and warming rates.

For preserving the shoot tips of in vitro and field grown blueberries, Dhungana et al., (2017) have attempted D- cryoplate method. The ideal explant, preculture treatments, loading and desiccation period were investigated and found that shoot tips from in vitro grown plants had better survival $(86.7 \%)$ than shoot tips from field grown current shoots $(59.7 \%)$. To induce osmo-tolerence, the shoot tips were precultured in MS media containing $0.3,0.5$ and $0.7 \mathrm{M}$ sucrose at $25{ }^{\circ} \mathrm{C}$ for varying durations of 1,2 and 3 days. Preculturing in MS media with $0.3 \mathrm{M}$ sucrose for one day was found better for higher regrowth $(79.5 \%)$ after cryopreservation.

The loading solution treatment ( $2 \mathrm{M}$ glycerol and $0.6 \mathrm{M}$ sucrose) of precultured shoot tips for 30 minutes followed by desiccation for one hour resulted in highest recovery of cryopreserved shoot tips in blueberry. Thus in vitro grown shoot tips precultured in $0.3 \mathrm{M}$ sucrose for one day, followed by loading with 2M glycerol and $0.6 \mathrm{M}$ glucose for 30 minutes at $25^{\circ} \mathrm{C}$ and desiccation for one hour was optimized for cryopreservation. With this protocol, cryopreservation of six blue berry cultivars using shoot tips from in vitro and current shoots was attempted. The percentage regrowth of in vitro shoot tips ranged from
46.7 to 100 per cent whereas in current shoot tips, recovery ranged from 17.2 to 71.5 percent. Highest regrowth was recorded in the cultivar Blugold (100\%) using in vitro grown shoot tips which was significantly higher than current shoot tips (17.2\%), followed by Festival (96.9\% in in vitro shoot tips). In cultivars viz., climax, Magnolia and Weymouth no significant difference was observed in regrowth percentage of in vitro and current shoot tips showing the influence of varietal response in cryopreservation (Dhungana et al., 2017).

Successful Cryopreservation of in vitrogrown shoot tips of strawberry by the vitrification method using aluminium cryoplates is reported by Yamamoto et al., (2012). In the present study, the shoot tips (1.5$2.0 \mathrm{~mm} £ 0.5-1.0 \mathrm{~mm}$ ) were dissected from the shoot and pre-cultured at $58 \mathrm{C}$ for $2 \mathrm{~d}$ on Murashige and Skoog medium containing 2M glycerol and $0.3 \mathrm{M}$ sucrose. The pre-cultured shoot tips were placed on the aluminium cryoplate containing ten wells embedded in alginate gel. Osmoprotection was performed by immersing the cryo-plates in a loading solution ( $2 \mathrm{M}$ glycerol and $0.8 \mathrm{M}$ sucrose) for $30 \mathrm{~min}$ at $25^{\circ} \mathrm{C}$. Dehydration was performed by immersing the cryo-plates in plant vitrification solution 2 for $50 \mathrm{~min}$ at $25^{\circ} \mathrm{C}$. Then, the cryo-plate with shoot tips was transferred into an uncapped cryotube that was held on a cryo-cane and directly immersed into liquid nitrogen (LN). After storage in LN, shoot tips attached to the cryoplate were directly immersed into $2 \mathrm{ml}$ of a $1 \mathrm{M}$ sucrose solution for regeneration. With this protocol, the regrowth levels of shoots of 15 strawberry cultivars were observed. Regrowth was very high for all cultivars, ranging from 70 to 97 per cent, with an average of 81 per cent for the 15 cultivars. Highest regrowth was recorded in Hatsukuni (97\%) followed by Cavalier (93\%). 


\section{Comparison of cryopreservation techniques}

There are advantages as well as disadvantages associated with each method. Information on efficacy of protocols is required before selecting the method of choice for long-term conservation of germplasm in gene banks. A study was conducted to compare the efficacy of existing methods namely simple freezing and vitrification with a fast freeze/fast-thaw (droplet freezing) method for meristems in banana by Agrawal et al., (2004). The average post-thaw shoot regeneration of the fast freeze/fast thaw protocol was 51.8 per cent and was superior to the existing protocols (12.5\%, 39.2\% and $42.8 \%$, respectively). In droplet freezing method, in addition to higher post-thaw regeneration, frequency of callus formation was lowest $(3.7 \%)$. It was found that the fast freeze/fast thaw technique was the best amongst all the four methods tested for the cryopreservation of in vitro banana meristems in the genotype Robusta.

\section{Stability of cryopreserved material}

Genetic stability of any plant material after $e x$ situ conservation, especially in case of in vitro and cryo gene banks are of important concern. Assessment of genetic stability in terms of morphological, histological and molecular integrity has earned greater attention since variations can occur due to various reasons such as tissue culture, toxicity due to cryoprotectants and regeneration process. Hence, before routinely establishing the cryobank any species, studies on the field performance and genetic fidelity of plants derived from cryopreserved plant material need to be carried out.

Agrawal et al., (2009) compared the performance of cryopreserved banana germplasm of cultivar 'Sommarani Monthan' (AAB, Monthan subgroup) for agronomic and molecular traits (using simple sequence repeats; SSR) with that of its parental type. Experiment included cryopreserved germplasm, cryopreservation control, in vitroconserved plants, and natural sucker raised plants.

Genetic stability of original mother plants maintained in the field genebank, in vitroconserved plants, PVS2-treated plants and cryopreserved plants was assessed using 12 agronomic traits and nine SSR primers. Results indicated that no significant differences existed for the major growth and yield parameters analyzed, except in one plant. Even the in vitro-conserved plants (conserved for 6 years) were statistically comparable to the original mother plants raised from natural suckers in all the important growth and yield parameters. In one of the plants raised from cryopreserved meristems, the fruit colour was found to be green instead of ash green. Among the nine primer pairs tested, seven primers pairs namely AGMI-33,34, AGMI-35,36, AGMI67,68, AGMI-93,94, AGMI-95,96, AGMI129,130 and MbSSR 1-149 amplified products resulting in discrete repeatable amplicons. All the alleles were found to be monomorphic. The SSR primer pairs tested also did not show any variation for the green fruited variant as compared with control plants

Choudhary et al., (2013) examined the genetic stability of cryopreserved dormant buds of Morus germplasm that were stored in liquid nitrogen using two-step freezing, then rewarmed and regrown. The plants were regenerated directly from dormant buds (before and after cryopreservation) without intermediary callus phase. Fifteen primers were selected for the RAPD analysis based on the reproducibility and banding patterns. All the primers have shown monomorphic banding patterns and did not reveal any polymorphism among the mother plant and in 
vitro regenerants before and after cryopreservation, suggesting that cryopreservation, using two-step freezing, does not affect genetic stability of mulberry germplasm.

\section{Status of cryopreservation in fruit crops}

Globally, successful cryopreservation protocols have been optimized for Banana, Pine apple, Citrus, Papaya, Strawberry, Japanese Persimmon, Olive, Date palm, Bluberry and Grapes using various explants adopting different cryogenic storage techniques. At the international, National Institute of Agro biological Resources (NIAR) of Japan holds about 50 accessions of mulberry and INIBAP, Laboratory of Tropical Crop Improvement, Belgium has 440 banana accessions in cryogenebank. At present, the institutes such as National Bureau of Plant Genetic Resources (NBPGR, New Delhi) and Indian Institute of Horticultural Research (IIHR, Bengaluru) are involved large-scale cryobanking of horticultural crops in India. The NBPGR holds 13363 accessions of various agri-horticultural species in the form of seeds/ embryo/ embryonic axes/ dormant buds/ meristems or pollen, of which 3465 accessions of fruits and nuts are cryopreserved as seeds, while IIHR has 675 accessions of pollen of various horticultural species (Agrawal et al., 2019).

In conclusion the cryopreservation protocols developed in various fruit crops can aid in long term conservation of genetic resources with several advantages of saving land, time, labour and energy without losing the maternal genetic fidelity. But the technique has its own demerits of using toxic chemicals, posing oxidative stress to the tissues, which needs to be investigated thoroughly. Though with manifold benefits, the cryogenic storage cannot be a standalone approach for conservation of fruit genetic resources, yet it can complement other conservation methods. Thus, it is expected that improved cryopreservation techniques will provide added security in the preservation of important woody plant germplasm formerly preserved only as field collections.

\section{References}

Agrawal, A., Sanayaima, R., Singh, R., Tandon, R., Verma, S., and Tyagi, R. K. 2014. Phenotypic and molecular studies for genetic stability assessment of cryopreserved banana meristems derived from field and in vitro explant sources. In Vitro Cell. Dev. Biol.-Plant 50(3): 345-356.

Agrawal, A., Singh, S., Malhotra, E. V., Meena, D. P. S., and Tyagi, R. K. 2019. In Vitro Conservation and Cryopreservation of Clonally Propagated Horticultural Species. In: Conservation and Utilization of Horticultural Genetic Resources, Springer, Singapore. pp. 529-578.

Agrawal, A., Swennen, R., and Panis, B. 2004. A comparison of four methods for cryopreservation of meristems in banana (Musa spp.). Cryoletters 25(2): 101-110.

Agrawal, A., Tyagi, R. K., Goswami, R., Uma, S., Saraswathi, M. S., and Durai, P. 2009. Cryo banking of banana (Musa sp.) germplasm in India: evaluation of agronomic and molecular traits of cryopreserved plants. In: International Symposium on Cryopreservation in Horticultural Species; Apr. 2009, Leuven. International Society of Horticultural Science, Leuven, pp.129138.

Choudhary, R., Chaudhury, R., Malik, S. K., Kumar, S., and Pal, D. 2013. Genetic stability of mulberry germplasm after cryopreservation by two-step freezing technique. African J. Biotech.12(41): 
5983-5993.

Dhungana, S. A., Kunitake, H., Niino, T., Yamamoto, S. I., Fukui, K., Tanaka, D., Maki, S., and Matsumoto, T. 2017. Cryopreservation of blueberry shoot tips derived from in vitro and current shoots using D cryo-plate technique. Plant Biotech. 34: 1-5.

Engelmann, F. 2004. Plant cryopreservation: progress and prospects. In Vitro Cell.

Dev. Biol. Plant 40(5): 427-433.

Fabre, J. and Dereuddre, J. 1990. Encapsulation-dehydration: a new approach to cryopreservation of Solanum shoot-tips. Cryoletters 11: 413-426.

Funnekotter, B., Bunn, E., and Mancera, R. L. 2017. Cryo-mesh: a simple alternative cryopreservation protocol. Cryo Letters 38(2): 155-159.

Gamez-Pastrana, R., Martinez-Ocampo, Y., Beristain, C. I., and Gonzalez-Arnao, M. T. 2004. An improved cryopreservation protocol for Pineapple apices using encapsulation-vitrification.

Cryoletters 25(6): 405-414.

Generoso, A. L., Carvalho, V. S., Walter, R., Campbell, G., da Silva Araújo, L., Santana, J. G. S., and da Cunha, M. 2019. Mature-embryo culture in the cryopreservation of passion fruit (Passiflora edulis Sims.) seeds. Sci. Hortic. 256(108638): 1-6.

González-Arnao, M. T., Ravelo, M. M., Villavicencio, C. U., Montero, M. M., and Englemann, F. 1998. Cryopreservation of Pineapple (Ananas comosus) apices. Cryoletters 19(6): 375-382.

Kalaiselvi, R., Rajasekar, M., and Gomathi, S. 2017. Cryopreservation of plant materials-a review. Int. J. Chem. Stud. 5(5): 560-564.

Kaya, E., Souza, F., Gökdoğan, E. Y., Ceylan, M., and Jenderek, M. 2017. Cryopreservation of citrus seed via dehydration followed by immersion in liquid nitrogen. Turkish J. Biol. 41(1): 242-248.

Matsumoto, T. 2017. Cryopreservation of plant genetic resources: conventional and new methods. Rev. Agric. Sci. 5: 13-20.

Matsumoto, T., Sakai, A., and Yamada, K. 1994. Cryopreservation of in vitrogrown apical meristems of wasabi (Wasabia japonica) by vitrification and subsequent high plant regeneration. Plant Cell Rep. 13(8): 442-446.

Merhy, T. S. M., Vianna, M. G., Garcia, R. O., Pacheco, G., and Mansur, E. 2014. Cryopreservation of Passiflora pohlii nodal segments and assessment of genetic stability of regenerated plants. Cryoletters 35(3): 204-215.

Niino, T., Yamamoto, S. I., Fukui, K., Martínez, C. R. C., Arizaga, M. V., Matsumoto, T., and Engelmann, F. 2013. Dehydration improves cryopreservation of mat rush (Juncus decipiens Nakai.) basal stem buds on cryo-plates. Cryoletters 34(6): 549-560.

Panis, B., Piette, B., and Swennen, R. 2005. Droplet vitrification of apical meristems: a cryopreservation protocol applicable to all Musaceae. Plant Sci. 168(1): 45-55.

Reed, B. M. 2017. Plant cryopreservation: a continuing requirement for food and ecosystem security. In Vitro Cellular and Developmental Biology-Plant, Springer, Singapore 53(4): pp.285-288.

Rohini, M. R., Malik, S. K., Choudhary, R., Kaur, S., Uchoi, A., and Chaudhury, R. 2016. Storage behavior and cryopreservation studies in Indian rough lemon (Citrus jambhiri): a promising rootstock for long-term conservation. Turkish J. Agric. and For. 40(6):865873.

Sakai, A. and Engelmann, F. 2007. Vitrification, encapsulation-vitrification 
and droplet-vitrification: a review. Cryoletters 28(3): 151-172.

Sakai, A., Kobayashi, S., and Oiyama, I. 1990. Cryopreservation of nucellar cells of navel orange (Citrus sinensis Osb. var. brasiliensis Tanaka) by vitrification. Plant Cell Rep. 9(1): 3033.

Schaefer-Menuhr, A., Schumacher, H. M., and Mix-Wagner, G. 1994. Long-term storage of old potato varieties by cryopreservation of meristems in liquid nitrogen. Landbauforschung Völkenrode 44: 301-313.

Uchendu, E. E., Muminova, M., Gupta, S., and Reed, B. M. 2010. Antioxidant and anti-stress compounds improve regrowth of cryopreserved Rubus shoot tips. In Vitro Cell. Dev. Biol. Plant. 46(4): 386-393.

Yamamoto, S. I., Fukui, K., Rafique, T.,
Khan, N. I., Martinez, C. R. C., Sekizawa, K., Matsumoto, T., and Niino, T. 2012. Cryopreservation of in vitro-grown shoot tips of strawberry by the vitrification method using aluminium cryo-plates. Plant Genet. Resour. 10(1):14-19.

Yamamoto, S. I., Rafique, T., Arizaga, M. V., Fukui, K., Gutierrez, E. J. C., Martinez, C. R. C., Watanabe, K., and Niino, T. 2015. The aluminum cryo-plate increases efficiency of cryopreservation protocols for Potato shoot tips. American J. Potato Res. 92(2): 250-257.

Yi, J. Y., Balaraju, K., Baek, H. J., Yoon, M. S., Kim, H. H., and Lee, Y. Y. 2018. Cryopreservation of Citrus limon (Burm). F shoot tips using a dropletvitrification method. Korean J. Plant Resour. 31(6): 684-694.

\section{How to cite this article:}

Anushma, P. L., K. Dhanyasree and Rafeekher, M. 2020. Cryopreservation of Fruit Genetic Resources-A Review. Int.J.Curr.Microbiol.App.Sci. 9(10): 1532-1542.

doi: https://doi.org/10.20546/ijcmas.2020.910.183 